\title{
EXTREMAL MINKOWSKI ADDITIVE SELECTIONS OF COMPACT CONVEX SETS
}

\author{
RADE T. ŽIVALJEVIĆ
}

(Communicated by William J. Davis)

\begin{abstract}
A function $f: \mathscr{K}^{n} \rightarrow R^{n}$, defined on the set of all compact convex sets in $R^{n}$, is a Minkowski additive selection, provided $f(K+L)=f(K)+$ $f(L)$ and $f(K) \in K$ for all $K, L \in \mathscr{K}^{n}$. The paper deals with selections which are extremal in some sense, in particular we characterize the set of all Minkowski additive selections which have the property $f(K) \in \operatorname{ext}(K)$ for all $K \in \mathscr{K}^{n}$, where $\operatorname{ext}(K)$ is the set of all extreme points of $K$.
\end{abstract}

\section{INTRODUCTION}

Let $\mathscr{K}^{n}$ be the set of all compact, convex sets in $R^{n}$ equipped with the topology generated by the Hausdorff metric. A vector valued valuation or shortly a valuation is a function $f: \mathscr{K}^{n} \rightarrow R^{n}$ (see [2]). A valuation $f$ is Minkowski additive if $f(K+L)=f(K)+f(L)$ for all $K, L \in \mathscr{K}^{n}$ and the class of all additive selections is the main object of study of this paper. Certainly, the most important and the best known valuation is the curvature centroid of a compact convex set $K$, first considered by J. Steiner (1840) in the case of a planar set. One of equivalent definitions, in the general case, is $s(K)=\int_{S^{n-1}} u h(K, u) d \nu(u)$ where $h(K, \cdot)$ is the support function of $K$ and $\nu$ is the normalized rotation invariant measure on $S^{n-1}$. Perhaps the best known result about Steiner points is the following remarkable result of $R$. Schneider [7], see also Posicel' skii [5], who confirmed a conjecture of Grünbaum by proving the following theorem.

Theorem 0. If $f: \mathscr{K}^{n} \rightarrow R^{n}$ is Minkowski additive, continuous and rigid motion equivariant then $f(K)$ is Steiner point of $K$.

In order to define the notion of an extreme additive selection and formulate the problem we are interested in, let us consider the set of all, not necessarily continuous, additive selections $\mathscr{L}=\left\{\varphi: \mathscr{K}^{n} \rightarrow R^{n} \mid\left(\forall K, L \in \mathscr{K}^{n}\right) \varphi(K+L)=\right.$ $\varphi(K)+\varphi(L) \& \varphi(K) \in K\}$. Let $\mathscr{L}$ be topologized as a subspace of $F\left(\mathscr{K}^{n}, R^{n}\right)$, the space of all functions from $\mathscr{K}^{n}$ to $R^{n}$ with the weak topology i.e. the

Received by the editors April 5, 1988.

1980 Mathematics Subject Classification (1985 Revision). Primary 52A20. 
weakest topology which makes the maps $F\left(\mathscr{K}^{n}, R^{n}\right) \rightarrow R^{n}, \varphi \mapsto \varphi(K)(K \in$ $\left.\mathscr{K}^{n}\right)$, continuous. Obviously, $\mathscr{L}$ is a compact, convex set in the locally convex, topological linear space $F\left(K^{n}, R^{n}\right)$ so one can talk about the set $\mathscr{E}$ of extreme points in $\mathscr{L}$. In light of the fact that every element of $\mathscr{L}$ is an average (we have in mind theorems of Krein-Milman and Choquet, see [3]) of its extreme points, it is desirable to characterize or to say as much as possible about $\mathscr{E}$.

\section{Regular extreme POINTS}

The problem of describing $\mathscr{E}$ in a useful way seems to be difficult so let us start with a more restricted class $\mathscr{R} \subset \mathscr{E}$ of so called regular extreme points. Precisely, $\mathscr{R}=\left\{\varphi \in \mathscr{E} \mid \varphi(K) \in \operatorname{ext}(K)\right.$ for all $\left.K \in \mathscr{K}^{n}\right\}$ where $\operatorname{ext}(K)$ is the set of all extreme points of $K$.

Theorem 1.1. There is a 1-1 correspondence between $\mathscr{R}$ and $O(n)$, the set of all orthogonal, linear transformations of $R^{n}$.

The proof of this theorem consists of two lemmas. Before they are formulated let us define the function $\pi: O(n) \rightarrow \mathscr{R}$ which will establish the 1-1 correspondence mentioned in the theorem. First, let us identify $O(n)$ with the set of all orthogonal frames, where an orthogonal frame, denoted by $\left[a_{1}, \ldots, a_{n}\right]$, is a set of $n$ mutually orthogonal unit vectors in $R^{n}$. For $u \in R^{n}$ let $\psi_{u}: \mathscr{K}^{n} \rightarrow \mathscr{K}^{n}$ be defined by $\psi_{u}(K)=K \cap\left\{z \in \mathscr{R}^{n} \mid\langle u, z\rangle=h(K, u)\right\}$ i.e. $\psi_{u}(K)$ is the intersection of $K$ with the supporting hyperplane orthogonal to $u$. $\psi_{u}(K)$ will be called the extremal face of $K$ in the direction of $u$. Let us note that $\psi_{u}(K+L)=\psi_{u}(K)+\psi_{u}(L)$. Indeed, if $h(K+L, u)=\alpha$, $h(K, u)=\beta, h(L, u)=\gamma$ then $\alpha=\beta+\gamma$ and $\psi_{u}(K+L)=\{x+y \mid x \in$ $K, y \in L,\langle u, x+y\rangle=\alpha\}=\{x+y \mid x \in K, y \in L,\langle u, x\rangle=\beta,\langle u, y\rangle=$ $\gamma\}=\psi_{u}(K)+\psi_{u}(L)$. If $g=\left[a_{1}, \ldots, a_{n}\right] \in O(n)$ let us define $\pi_{g} \in \mathscr{R}$ by $\pi_{g}(K)=\psi_{a_{n}} \circ \psi_{a_{n-1}} \circ \cdots \circ \psi_{a_{1}}(K)$. Since $\psi_{u}(K)$ lies in a hyperplane orthogonal to $u$, we see that $\pi_{g}(K)$ is a single point whereas the additivity of $\pi_{g}$ follows from the additivity of $\psi_{a_{i}}, 1 \leq i \leq n$. Since obviously $\pi_{g}(K) \in \operatorname{ext}(K)$ we have just established the following lemma.

Lemma 1.1. For every $g \in O(n), \pi_{g}$ is a regular extreme point.

$\pi$ is obviously a 1-1 map so it remains to be shown that it is onto.

Lemma 1.2. For every $\varphi \in \mathscr{R}$ there exists $g \in O(n)$ such that $\varphi=\pi_{g}$.

Proof. Let $V_{1}=\left\{x \in R^{n} \mid\|x\| \leq 1\right\}$ be the unit ball and $a_{1}=\varphi\left(V_{1}\right)$. If $V_{2}=$ $\left\{x \in V_{1} \mid\left\langle x, a_{1}\right\rangle=0\right\}$ let $a_{2}=\varphi\left(V_{2}\right)$. Inductively, if $a_{i} \in R^{n}$ and $V_{i} \subset R^{n}$ are defined, $i<n$, let $V_{i+1}=\left\{x \in V_{i} \mid\left\langle x, a_{i}\right\rangle=0\right\}$ and $a_{i+1}=\varphi\left(V_{i+1}\right)$. Since all $a_{i}, 1 \leq i \leq n$, are unit vectors by the regularity of $\varphi$, and mutually orthogonal by the construction, let $g=\left[a_{1}, \ldots, a_{n}\right] \in O(n)$. We claim that $\varphi(K)=\pi_{g}(K)$ for every $K \in \mathscr{K}^{n}$. First of all this is true for all sets $V_{1}, V_{2}, \ldots, V_{n}$ by the construction. Now, let us suppose $\varphi(K) \neq \pi_{g}(K)$ for some $K \in \mathscr{K}^{n}$. Further, let $K_{i}, 0 \leq i \leq n$, be defined inductively by $K_{0}:=K, K_{i+1}=\psi_{a_{i+1}}\left(K_{i}\right)$, 
$0 \leq i \leq n-1$, so in particular $K_{n}=\left\{\pi_{g}(K)\right\}$. Obviously, there exists $0 \leq i<n$ such that $\varphi(K) \in K_{i} \backslash K_{i+1}$. Since $\varphi\left(K+m V_{i+1}\right)=\varphi(K)+m \varphi\left(V_{i+1}\right), m \in N$, we observe that for $m$ big enough $\varphi\left(K+m V_{i+1}\right)$ is in the relative interior of the set $\pi_{g}(K)+m V_{i+1} \subset K+m V_{i+1}$ which contradicts the assumption $\varphi(L) \in \operatorname{ext}(L)$ for all $L \in \mathscr{K}^{n}$. Hence $\varphi(K)=\pi_{g}(K)$ for all $K \in \mathscr{K}^{n}$.

Let us note that $\pi_{g}$ cannot be continuous for any $g \in O(n)$. This can be seen directly but we prefer to prove a more general result.

Proposition 1.1. If $f: \mathscr{K}^{n} \rightarrow R^{n}$ is a continuous selection then $f(K)=s(K)$ for some $K \in \mathscr{K}^{n}$.

Proof. Actually the following proof shows that the equality $f(K)=s(K)$ holds quite often. Let $K \in \mathscr{K}^{n}$ be a centrally symmetric convex body such that $s(K)=0$. Let $\mathfrak{G}$ be the Grassman manifold of all hyperplanes containing the origin and $\xi: E \stackrel{p}{\longrightarrow} \mathfrak{G}$ the canonical $(n-1)$-plane bundle over $\mathfrak{G}$, i.e. $E=\{(l, v) \mid v \in l\} \subset \mathfrak{G} \times R^{n}$. It is well known that this bundle cannot have a nowhere zero section which follows from the fact that the total Stiefel-Whitney class of this bundle has the form $w(\xi)=1+u+\cdots+u^{n-1}, u \in H^{1}\left(\mathfrak{G}, Z_{2}\right)$, i.e. this bundle does not split since the total Stiefel-Whitney class is multiplicative and $w(\xi)$ has a nonzero $u^{n-1}$-term (see [4] for details). $\mathfrak{G}$ can be embedded in $\mathscr{K}^{n}$ by the map $I: \mathfrak{G} \rightarrow \mathscr{K}^{n}, l \mapsto l \cap K$, which induces a bundle $\xi^{\prime}: E^{\prime} \rightarrow I(\mathfrak{G})$ isomorphic to $\xi$. Since $s(l \cap K)=0$ for all $l \in \mathfrak{G}$ ( $K$ is centrally symmetric) we see that $f \uparrow I(\mathfrak{G})$ can be thought of as a section of this bundle so in particular $f(l \cap K)=0$ for some $l \in \mathfrak{G}$.

Let us observe that $\pi: O(n) \rightarrow \mathscr{R}$ is not continuous either. Otherwise the map $O(n) \stackrel{\pi}{\longrightarrow} \mathscr{R} \underset{\operatorname{ev}(\cdot, K)}{\longrightarrow} \operatorname{ext}(K)$, where $\operatorname{ev}(\varphi, K)=: \varphi(K)$, for a fixed polyhedron $K$ would be onto and continuous which is a contradiction with the finiteness of $\operatorname{ext}(K)$. On the other hand the map $\operatorname{ev}(\cdot, K) \circ \pi: O(n) \rightarrow R^{n}$ is measurable for every $K \in \mathscr{K}^{n}$. This follows from the fact that the set $A=\left\{e \in S^{n-1} \mid\left(\exists_{1} u \in K\right) h(K, e)=\langle u, e\rangle\right.$ has $\nu$-measure 1 where $\nu$ is the normalized, rotation invariant measure on $S^{n-1}$. Let $\mu$ be the normalized Haar-measure on the group $O(n)$ and let $\alpha: O(n) \rightarrow S^{n-1}$ be the map $\left[a_{1}, \ldots, a_{n}\right] \mapsto a_{1}$. Then the following holds.

Proposition 1.2. $s(K)=\int_{O(n)} \pi_{g}(K) d \mu(g)$.

Proof. The proof immediately follows from Theorem 0. Alternatively, one can directly prove, first for smooth $K$, that

$$
\int_{O(n)} \pi_{g}(K) d \mu(g)=\int_{S^{n-1}} u h(K, u) d \nu(u)
$$

using the fact that $\pi_{g}$ is $\gamma$-a.e. constant function along fibers of $\alpha$ and then extend the result by continuity. The last proposition is interesting because it says that, in order to give a Choquet [3] representation of Steiner selection $s$ 
it is possible to concentrate the representing measure on the set $\mathscr{R}$ of regular extreme points.

\section{NONREGULAR EXTREME POINTS}

Can it be that $\mathscr{E}=\mathscr{R}$ ? In light of examples of R. Schneider [8], pp. 76 and G. T. Sallee [6] it is very likely that the following conjecture holds.

Conjecture. $\mathscr{E} \backslash \mathscr{K} \neq \varnothing$. A natural way to establish the conjecture above is following. One has to find a valuation $\varphi \in \mathscr{L}$ and a continuous linear functional $\Lambda: F\left(\mathscr{K}^{n}, R^{n}\right) \rightarrow R$ such that $\Lambda(\varphi)>\Lambda\left(\pi_{g}\right)$ for all $g \in O(n)$. It is not diffcult to see, since $F\left(\mathscr{K}^{n}, R^{n}\right)$ is a topological product of 1-dimensional spaces, that every continuous linear functional has the form $\Lambda(\varphi)=\left\langle\varphi\left(K_{1}\right), v_{1}\right\rangle+\cdots+$ $\left\langle\varphi\left(K_{r}\right), v_{r}\right\rangle$ for some choice of $K_{1}, \ldots, K_{r} \in \mathscr{K}^{n}$ and $v_{1}, \ldots, v_{r} \in R^{n}$. Now, a natural choice for $\varphi \in \mathscr{L} \backslash \mathrm{cl}(\operatorname{conv} \mathscr{R})$ could be either the valuation defined by Sallee or the one defined by Schneider but we do not know if either of them can be a selection, i.e. whether $\varphi(K) \in K$ holds for all $K \in \mathscr{K}^{n}$.

\section{REFERENCES}

1. B. Grünbaum, Measures of symmetry for convex sets, Proc. Sympos. Pure Math., vol. 7, Amer. Math. Soc., Providence, R.I., 1963, pp. 233-270.

2. P.McMullen and R. Schneider, Valuations on convex bodies, Convexity and its Applications, Birkhauser, 1983.

3. P. A. Meyer, Probability and potentials, Blaisdell Publ. Company, 1966.

4. J. Milnor and J. D. Stasheff, Characteristic classes, Princeton Univ. Press. Princeton, N.J., 1974.

5. E. D. Posicel' skii, Characterization of Steiner points, Mat. Zametki 14 (1973), 243-247. (Russian)

6. G. T. Sallee, A non-continuous "Steiner point", Israel J. Math. 10 (1971), 1-5.

7. R. Schneider, On Steiner points of convex bodies, Israel J. Math. 9 (1971), 241-249.

8. __ Equivariant endomorphisms of the space of convex bodies, Trans. Amer. Math. Soc. 194 (1974), 53-78.

Matematički Institut, KNez Mihailova 35/1, 11000 Yugoslavia 